based on the major capsid protein, L1, which self assembles into virus-like particles. Although HPV L1 is a relatively conserved gene with different HPV types having L1 sequence homology of up to $90 \%$, the vaccine induced protection is HPV type-specific. Both vaccines induce excellent protection against the specific types targeted; however, there is also limited protection from infection with closely related HPV types. The vaccines do not cause regression of established genital lesions: regression is due to cell-mediated immunity. The serological correlates of protection of the vaccines are not defined but it is likely that protection is largely due to neutralising antibodies: animal studies have shown that protection can be transferred by passive immunisation with immune sera. In natural infections, titres of HPV antibodies are much lower than for vaccine induced antibodies, and in fact not all infected people develop detectable antibodies. It is likely that serological correlates of protection will become better defined only if vaccine induced protection starts to wane as the antibody titres potentially drop over time; it may then be possible to define levels of antibody needed for protection. Such information could inform the need for further booster vaccinations.

\section{S12.2 TALES OF WOE: HSV VACCINE AND NULL STUDY RESULTS}

doi:10.1136/sextrans-2013-051184.0058

\section{A Wald. University of Washington, Seattle, WA, United States}

Herpes simplex virus type 2 (HSV-2) infects 530 million people, is the predominant cause of genital ulcer disease, and a key driver of HIV epidemic in subSaharan Africa. In human populations with susceptibility to HSV appears universal. In animal models, several candidate preventative and therapeutic vaccines have been successful only to fail in clinical trials, including recent null results from the prophylactic glycoprotein D2 subunit vaccine trial. However, detailed analyses of the immune suggest assays that antibody response may correlate with protective immune response, providing the first evidence of immune correlate of protection from infection. In addition, recent characterization of CD8 tissue memory cells in genital mucosa suggests that we may also identify immune correlate of HSV-2 control in the infected host. In this Symposium, I will discuss HSV-2 vaccine efforts in the context of our understanding of pathogenesis and epidemiology, as well as the current pipeline of candidate vaccines.

\section{S12.3 UNDERSTANDING HOW BROADLY CROSS-NEUTRALISING ANTIBODIES DEVELOP IN HIV INFECTION - CLUES FOR HIV VACCINE DEVELOPMENT?}

doi:10.1136/sextrans-2013-051184.0059

L Morris. National Institute for Communicable Diseases, Johannesburg, South Africa

Neutralizing antibodies are likely to play a crucial role in a preventative HIV-1 vaccine. Although efforts to elicit broadly cross-neutralising (BCN) antibodies by vaccination have been unsuccessful, a minority of individuals naturally develops these antibodies after many years. Thus understanding how these antibodies evolve could provide a template for HIV vaccine design. To date we have studied $\sim 80$ HIV-infected individuals in the long-term CAPRISA cohort and found that approximately a quarter develop cross-neutralising antibodies by 3 years of infection. Analysis of longitudinal samples showed that breadth developed gradually starting from year 2 , with the number of viruses neutralised as well as the antibody titer increasing over time, peaking at 4 years post-infection with little activity thereafter. The extent of cross-neutralising activity correlated with CD4 $\mathrm{T}$ cell decline and viral load at 6 months post infection, suggesting that early events set the stage for the development of breadth. Mapping of the epitopes targeted by cross-neutralising antibodies revealed that in most cases they recognised one of the 4 well-defined sites of vulnerability on the HIV envelope. This included the two glycan-dependent epitopes in the V2 and C3 regions of gp120, the CD4 binding site as well as the membraneproximal external region (MPER). Isolation of monoclonal antibodies from some of these individuals has confirmed the plasma specificities and revealed interesting insights into antibody ontogeny. Furthermore, we have shown how viral escape from earlier strain-specific antibodies contributes to the formation of neutralising antibody epitopes highlighting the dynamic interplay between viral and antibody evolution. Our ongoing work aims to more precisely define the changes in the antibody and viral repertoires that accompany the development of broadly cross-neutralising antibodies with the aim of mimicking these events in a vaccine scenario.

\section{S12.4 CHLAMYDIA TRACHOMATIS}

doi:10.1136/sextrans-2013-051184.0060

R M Johnson. Indiana University School of Medicine, Indianapolis, IN, United States

In vaccine enterprises there are two goals that must be achieved to be successful. Priority is usually given to the vaccine components, which antigen(s) and adjuvants. However an equally important goal is to understand the parameters of protective immunity, or the immunologic goal of vaccination. Chlamydia trachomatis infections pose a unique challenge for host immunity. In the majority of infected individuals the invading bacteria replicate only in the epithelial monolayer lining the reproductive tract. The business end (mechanism) of an effective vaccine is likely to be $T$ cells functioning at the epithelial interface. During natural infections individuals' immune responses range from asymptomatic clearance, to asymptomatic infertility, to hospitalisation for PID. Early Chlamydia vaccine attempts were associated with enhanced immunopathology. It is possible that Chlamydia vaccine candidates will cause enhanced immunopathology, with or without enhanced protection from bacterial replication in the reproductive tract. Evaluation of Chlamydia vaccines will need to include their ability to limit bacterial replication and immunopathology. There are some after-the-fact correlates for bad outcomes in women infected with $C$. trachomatis including circulating peripheral mononuclear cells that make too little IFN- $\gamma$, too much IL-10 or TNF $\alpha$ when activated by $C$. trachomatis antigens. The Chlamydia muridarum mouse model provides an opportunity to define the parameters of protective immunity in the genital tract, and then evaluate/validate them in humans. The mouse model has shown the CD4 T cells are critical to protection and CD8 T cells likely responsible for immunopathology. More recently it was demonstrated that there are redundant mechanisms for clearing C. muridarum from the genital tract. This presentation will cover the newest mouse model data, correlating it with what is known in humans. The practicable human correlates of protective immunity and immunopathology are likely to be novel CD4 and CD8 $T$ cell subsets, with antibody possibly falling into disfavour.

\section{S.13 - Challenges to a comprehensive approach to the prevention of HIV and STI among men who have sex with men in Europe (organised by ECDC)}

\section{S13.1 CURRENT AND FUTURE CHALLENGES IN GAY MEN'S HEALTH}

doi:10.1136/sextrans-2013-051184.0061

\section{G J Hart. University College London, London, UK}

Epidemics of HIV in men who have sex with men (MSM) were first recognised in the early 1980s. Since the introduction of antiretroviral treatment (ART) in the mid-1990s incidence of HIV has not, despite 